\title{
Neuronal morphology alterations in autism and possible role of oxytocin
}

\author{
Hisham El Falougr ${ }^{1}$, Barbora Filova ${ }^{1}$, Daniela Ostatnikova ${ }^{1}$, Zuzana Bacova ${ }^{2}$, Jan Bakos ${ }^{1,2}$ \\ ${ }^{1}$ Faculty of Medicine, Comenius University, Bratislava, Slovakia; ${ }^{2}$ Institute of Experimental Endocrinology, Biomedical \\ Research Center, Slovak Academy of Sciences, Bratislava, Slovakia \\ E-mail: j.bakos@savba.sk
}

\begin{abstract}
Current understanding of the neuroanatomical abnormalities in autism includes gross anatomical changes in several brain areas and microstructural alterations in neuronal cells as well. There are many controversies in the interpretation of the imaging data, evaluation of volume and size of particular brain areas, and their functional translation into a broad autism phenotype. Critical questions of neuronal pathology in autism include the concept of the reversible plasticity of morphological changes, volume alterations of brain areas, and both short- and long-term consequences of adverse events present during the brain development. At the cellular level, remodeling of the actin cytoskeleton is considered as one of the critical factors associated with the autism spectrum disorders. Alterations in the composition of the neuronal cytoskeleton, in particular abnormalities in the polymerization of actin filaments and their associated proteins underlie the functional consequences in behavior resulting in symptoms and clinical correlates of autism spectrum disorder. In the present review, a special attention is devoted to the role of oxytocin in experimental models of neurodevelopmental disorders manifesting alterations in neuronal morphology.
\end{abstract}

Key words: oxytocin, autism, brain anatomy, structural neuronal changes

Morphological and structural changes in neuronal cells are often associated with pathological conditions in the brain. Overall, it is becoming clear that disruptions in the neuronal cells development result in more or less visible neuroanatomical abnormalities present in the neurodevelopmental disorders including autism. Autism is a disorder characterized by impairments in the social interaction, language, behavior, and cognitive functions (Rapin and Katzman 1998; Brambilla et al. 2003). The autism phenotype is not identical across the affected persons. The heterogeneity of the disease progression, the severity of its symptoms, and the various co-morbid associated disorders leads to a belief of the variable neuropathological substrates of the disease.

Moreover, there is an ongoing debate that how much permanent are the neuromorphological alterations in the brain and whether there exists a reversible plasticity of such changes. Many research activities have been devoted to the critical periods in the development when the brain is the most sensitive to various environmental factors. The core features and signs of autism provide evidence about the affected brain areas and the involved neural systems (Amaral et al. 2008; Nickl-Jockschat et al. 2012). In this review, we focus on two major morphological features related to autism spectrum disorders. First, the gross anatomy differences in the various brain structures are described in relation to the distinct autistic behavioral phenotypes and second, available data at the level of cellular and molecular abnormalities in neuronal cells are gathered in association with symptoms and clinical correlates of autism spectrum disorder. Special attention is devoted to the role of oxytocin in experimental mouse and rat models of neurodevelopmental disorders manifesting alterations in neuronal morphology and humans.

Corresponding author: Jan Bakos, PhD., Biomedical Research Center, Institute of Experimental Endocrinology, Slovak Academy of Sciences, Dubravska cesta 9, Bratislava, Slovakia; phone: +421-2-3229 5232; e-mail: j.bakos@savba.sk. 


\section{The brain pathology in autism: overgrowth or degeneration?}

Magnetic resonance imaging (MRI) and postmortem tissue studies have been used in searching for the neuroanatomical substrate of autism (Piven et al. 1990). These two approaches have helped in understanding of the macroscopical and microscopical abnormalities in the autistic brain at different age (Schumann and Nordahl 2011; Donovan and Basson 2017). Anatomical abnormalities have already been found during the first three postnatal years, although brain volume changes were not observed in all children suffering of autism (Zielinski et al. 2014). MRI scans performed on a large sample of individuals has provided evidence that the brain of the autistic children is larger than in the normal children. In the adulthood, the brain size was normal or even smaller (Courchesne et al. 2011a). Recently, an extensive meta-analysis has been performed in the autism and different measures of the cerebral anatomy have been done (Traut et al. 2018).

Nevertheless, no conclusive result regarding the volume and size of the brain structure has been drawn. However, the factors like age and sex, as essential sources of the variability, have been stressed. There exists a considerable variation within human studies in the neuroanatomical abnormalities present in autism. Therefore, conclusions have to be drawn very carefully as many conflicting results have been published. Recently, using MRI scans, presence of cortical neuroanatomical abnormalities in autistic patients has been revealed (Yang et al. 2016). These authors have evaluated multiple morphological measures, such as cortical thickness, surface area, and cortical volume (Yang et al. 2016). From this perspective, microstructural or cellular changes could not be detected. Imaging studies, however, have brought other numerous data related to the autism as it will be discussed below.

Increasing number of studies have described an abnormal cellular growth and organization of autistic individuals brains (Pearson et al. 2013). Pearson et al. (2013) have focused on the extracellular matrix and they have found reduced levels of glycosaminoglycan and higher cell proliferation in particular subventricular zone. Thus, cellular defects in autism could be represented by brain enlargement. One study has also shown abnormal excess in a number of neurons in postmortem prefrontal cortex of autistic patients using stereological methods (Courchesne et al. 2011b). There is an ongoing discussion how the neuronal overgrowth may be translated into the clin- ical features of the disorder. On the other hand, another recent study has suggested that there could be a degenerative component present in the autism spectrum disorder (Avino et al. 2018). On the basis of the stereological analysis, a reduction in the number of amygdala neurons has been demonstrated (Avino et al. 2018). Another study of Wegiel et al. (2014) has also shown significant volume reductions in various subcortical brain structures in the autistic subjects. The above mentioned authors have interpreted their finding by the presence of early childhood and delayed neuronal growth during adolescence in autism. Head circumference and weight have also been studied in a sample of autistic patients in Norwegian population, and the results have confirmed that throughout the first year, the head circumference of girls with autism spectrum disorder was reduced in comparison with the non-autistic ones (Suren et al. 2014). It could be essential to pay more attention to the size of the individual brain areas. Indeed, numerous studies are analyzing the neuroanatomical alterations in autism using imaging techniques.

\section{Imaging of brain areas involved in the development of autism}

The components of the nervous system that underlie autism spectrum disorder most likely include the frontoparietal and frontotemporal areas, amygdalahippocampal complex, cerebellum, basal nuclei, and anterior and posterior cingulate areas (DeRamus and Kana 2014; Ecker et al. 2015). One of the prominent theories of the neuropathology of autism indicates that the brain undergoes a period of increased growth during early postnatal life succeeded by a deceleration in age-related growth (Amaral et al. 2008). Results of numerous MRI studies have indicated larger brain volume in children with autism spectrum disorders (ASD) of age 2-4 years than typically developing children (Carper and Courchesne 2005; Courchesne et al. 2011 a; Ecker et al. 2015). The increased brain volume disappears around the age of 6-8 years, afterward no considerable increase in the total brain volume commonly occurs (Carper and Courchesne 2005). Altered brain neurodevelopment in the autistic patients appears to differ across the diverse brain areas, with the frontal and temporal lobes being affected more than the parietal and occipital ones (Courchesne et al. 2011a; Ecker et al. 2015). Several studies have reported an enlargement in the cerebral gray and white matter in the frontal, parietal, and temporal lobes in the autism individuals. The most consistent increase has been shown in the frontal lobe. Abnormalities in 
cortical shape have been recognized in the Sylvian fissure, the superior temporal sulcus, the intraparietal sulcus, and the inferior frontal gyrus. Atypical cortical gyrification in individuals with autism has been described in many MRI studies (Piven et al. 1990; Auzias et al. 2014; DeRamus and Kana 2014; Ecker et al. 2015) including defects such as polymicrogyria, schizencephaly, and macrogyria (Ecker et al. 2015). Lack of empathy, difficulty in relating to others, and recognizing their emotion are characteristic signs for the autism individuals (Gillberg 1992; Hadjikhani et al. 2006). A possible neural substrate of empathy is the mirror neuron system, which has been found to be composed of a network of areas in the human brain. This network includes the pars opercularis of the inferior frontal gyrus, the inferior frontal cortex, the superior temporal sulcus, and the inferior parietal lobule. A local decrease in the gray matter in autism individuals has been reported in regions belonging to the mirror neuronal system (Gallese 2003; Leslie et al. 2004). The gray matter thinning has also been detected in areas involved in the emotion recognition and the social cognition. The cortical thinning of the mirror neuronal system has been associated with a severity of the autistic signs (Hadjikhani et al. 2006). The corpus callosum is the major commissural white matter in the brain. It is connecting the homologous and heterotopic areas of the cerebral hemispheres, therefore integrates the sensory, motor, and higher cognitive functions of the brain (Paul et al. 2007). Three-dimensional volumetric measurements of the corpus callosum in several studies have shown a reduction in the overall structure or one or more components of this commissural pathway. The alteration of the corpus callosum is considered to be the anatomical substrate of the processing and integration deficit in autism (Bellani et al. 2013a; Kucharsky Hiess et al. 2015). Inconstant abnormalities of the caudate and lenticular nuclei have been reported in a few MRI studies. The volume of the caudate nucleus is believed to be correlated with the ritualistic-repetitive behaviors in adolescents and adults individuals with autism (Brambilla et al. 2003; Amaral et al. 2008). Neuroanatomical abnormalities have been reported in the limbic system in the autistic subjects. The limbic structures are essential in sensorimotor gating and altering. It may partly explain the failure of subjects with autistic disorder to inhibit repetitive thoughts and actions (McAlonan et al. 2002). An increased cell packing density and decreased cell size has been observed in the amygdala, the entorhinal cortex, the mammillary body, the anterior cingulate gyrus, the medial septal nucleus, and the hippocam- pal formation (Raymond et al. 1996). Decreased hippocampal measures have been found in children and adult subjects with autism in MRI studies when compared to age-matched controls (Brambilla et al. 2003). Amygdala is a relatively small subcortical structure located in the temporal lobe and included into the limbic system. Its functions include the monitoring of the environment for possible danger and modulating levels of vigilance and controlling social behavior. Accordingly, the researchers have hypothesized the involvement of the amygdala into the socio-emotional impairment in the autistic individuals (Baron-Cohen et al. 2000). However, the investigations performed on amygdala produced conflicting results. Enlarged, reduced, and preserved volume has been reported in the studies on the amygdala morphology (Stanfield et al. 2008; Schumann et al. 2009; Bellani et al. 2013b). This difference of the results was explained on the base of age-related effects on the amygdala volume. Autistic toddlers and children frequently show a bilateral increase of the amygdala volume relative to age-matched controls. Volume reduction or preserved size of the amygdala has been reported in older adolescents and adults (Stanfield et al. 2008; Bellani et al. $2013 \mathrm{~b})$. The cerebellum is traditionally involved in the performance of the precise motor behavior, besides the growing evidence of its involvement in cognitive and affective functions. Pathological changes in the cerebellum in individuals with ASD may be the background of the varying degree of dyspraxia and alteration of the cognitive and affective functions (Traut et al. 2018). Several MRI studies have reported increased total, gray, and white matter volumes in the cerebellum of children and young adults with autism (Abell et al. 1999; Courchesne et al. 2001; Traut et al. 2018). In contrast to the total volume of the cerebellum, the size of the vermis seems to be slightly smaller in some subjects with autism (Kaufmann et al. 2003).

\section{Long-lasting changes or short-term effects?}

As described above, neuroanatomical abnormalities reported in various brain areas are often complex and lifelong. Although it is known that the shape and geometry of neuronal cells are dynamically changing in response to different internal and environmental stimuli, their alterations could become permanent and thus affecting the functionality of neuronal circuits for a long time. The concept of specific anatomical abnormalities present in autism is still under debate (Haar et al. 2016), although doubts have been raised on distinct brain regions. The lack of consistent anatomical data could be due to heterogeneous 
diagnosis criteria, inclusion and exclusion criteria, variability in age of participants and differences in imaging techniques in various studies. No apparent alterations in gross brain structure or hippocampal cytoarchitecture have been found in the mouse model of autism based on a deficiency of the SHANK3 gene (Bozdagi et al. 2010).

On the other hand, these authors rather point to the alterations in structural plasticity of synapses. Nevertheless, short-term changes of neuronal morphology play a role, in particular in the formation of the synaptic connections and functionality of neural networks (Nagel et al. 2012; Pani et al. 2013). Well-defined neuronal networks dynamically develop, especially in the relation to the regulation of the growth of neuronal projections and branching of the dendritic trees. Time-lapse imaging of dendritic spines allows evaluating motility of dendritic spines in hours (Verkuyl and Matus 2006). In this context, it is important to emphasize that recent studies even go to the level of the nanomolecular architecture of synaptic proteins (Hruska et al. 2018). Dynamics of the actin cytoskeleton is very high. One study has shown that a large amount of actin is exchanged over a period of minutes in dendritic spines (Star et al. 2002). Regulation of this turnover could be particularly affected in the neurodevelopmental disorders. It implicates that short-term multistep mechanisms underlying neuritogenesis, axon pathfinding, and dendritic spine motility, could be associated with autism spectrum disorders (Bakos et al. 2015).

\section{The diversity of neuronal cells}

Neuronal morphology largely depends on the type of neuronal cell and its locality in the specific brain region. Therefore, in the view of better understanding the diversity of cell types across multiple nervous systems, free accessible databases containing electrophysiological, morphological, and transcriptomic data have been established (http://celltypes.brainmap.org/, http://www.neuromorpho.org/). Improvements in high definition visualization bring new and tridimensional data on the dendritic and axonal morphology of single neurons (Peng et al. 2015). Morphological properties of neurons and glial cells are very complex between them the size, volume, diameter, and branching pattern as well (Comin and da Fontoura Costa 2013). Accumulated evidence suggest astrocytes abnormalities in white matter in autism. The increased levels of glial fibrillary acidic protein (GFAP) in the anterior cingulate cortex has been observed in males with ASD (Crawford et al. 2015).
On the other hand, the pathophysiological study of astrocyte density and morphology in the white matter of the dorsolateral prefrontal cortex has confirmed no significant differences (Lee et al. 2017). Also, the volume of white matter in ASD can be abnormally enlarged. The elevated volume of periventricular white matter hypointensity $(\mathrm{WMH})$ has been found in children with ASD. However, this abnormality was not associated with age. WMH volume of young adults was not changed in comparison with children (Blackmon et al. 2015). The growth of neuronal cells is accompanied with the expansion of cell membranes and rearrangement of the neuronal cytoskeleton. As a consequence, neurons elongate their extensions and form synaptic connections. It is well known that reorganization of the neuronal cytoskeleton plays a role in the deficits in various cognitive and motor functions (Gordon-Weeks and Fournier 2014). It is likely that dysregulation of the signaling pathways responsible for rearrangement of the neuronal cytoskeleton contributes to the development of autistic-like behavior.

\section{Actin cytoskeleton}

Remodeling of the actin cytoskeleton is considered as one of the critical factors associated with the autism spectrum disorders (Joensuu et al. 2018). Alterations in the composition of neuronal cytoskeletal in particular abnormalities in the polymerization of actin filaments and their associated proteins have been observed in the mouse strain C58/J model of autism (Baron-Mendoza et al. 2018). In the model of autism, it has been demonstrated that SHANK 3 deficient mice suffer from a loss of cortical actin filaments, in association with reduced Rho-GTPase activity together with increased activity of cofilin (Duffney et al. 2015). Indeed, accumulating a number of recent studies brought evidence that disruption of the actin cytoskeleton through dysregulated Rho GTPases contributes to autistic phenotype (Zeidan-Chulia et al. 2013; Sadybekov et al. 2017). It has been demonstrated that abnormal control of actin polymerization could be implicated in the regulation of glutamatergic synapses in autism spectrum disorder (Sadybekov et al. 2017). Rho-family of GTPases can affect dendritic spine development through regulation of the filamentous actin. We have already suggested that abnormalities in interactions of Rho GTPases with scaffolding proteins and actin cytoskeleton contribute to neurodevelopmental disorders (Reichova et al. 2018). In addition, various studies have found that the autistic symptoms can be 
rescued by manipulating actin regulators in SHANK 3 or FMR1-deficient mice (Duffney et al. 2015; Joensuu et al. 2018).

\section{Oxytocin role in neuronal morphology}

Anatomical and morphological changes of neuronal cells in the adult central nervous system under the influence of oxytocin are not representing a new idea. The brain plasticity either during early development or in adult age is well known, and many factors come into the play. In general, reorganization of the nervous tissue depends on various neuropeptides including oxytocin (Bakos et al. 2016). Oxytocin can affect multiple molecular and cellular aspects of neuronal cells ranging from excitatory and inhibitory properties of synapses to rearrangement of the actin cytoskeleton (Leonzino et al. 2016; Lestanova et al. 2016; Zatkova et al. 2018). We have demonstrated that oxytocin increases the expression of cytoskeletal proteins associated with neuronal growth (Lestanova et al. 2016). Effect of oxytocin on the cytoskeleton is not surprising as other studies have also shown that oxytocin promotes the formation of filamentous actin in the hypothalamic cells (Wang and Hatton 2006). Wang and Hatton (2006) have described spatiotemporal changes in the distribution of actin fibers in neuronal cells. It is important to state that mentioned effects are short-term. It is also known that actin dynamics represents fast, transient, and reversible cycles of polymerization and depolymerization of actin (van Goor et al. 2012). Therefore, it could be tricky to interpret some results related to oxytocin action on the cytoskeleton. Another study by Wang and Hatton (2007) has found that oxytocin has two phases of action. Initially increases the polymerization of F-actin and then decreases it after $30 \mathrm{~min}$. These authors explain their findings by potential interference with cofilin, which could severe actin fibers after a prolonged time. Nevertheless, the effects of oxytocin on neuron morphology are likely associated with the actin and the actin-binding proteins.

\section{Mechanisms of oxytocin action on neuronal morphology}

Structural effects of oxytocin on neurons and glial cells were described in the 1980s by Theodosis and Poulain (1984). At the very beginning, changes in the ultrastructural reorganization of the hypothalamic cells were associated with adaptations to lactation. However later, they were extended to other physiological processes including adaptations in response to os- motic stimulation (Chapman et al. 1986). Since then, several observations have proposed that oxytocin is involved at a certain degree to the rearranging of nervous tissue. Short-term effects of oxytocin in the rat hypothalamic $\mathrm{H} 32$ cells has suggested that mitogenactivated protein kinases play a role in morphological changes of neuronal cells (Meyer et al. 2018).

Moreover, recent studies have suggested that perinatal exposure to oxytocin has neurodevelopmental consequences (Palanisamy et al. 2018). It has been discovered that oxytocin promotes the survival and maturation of newborn pyramidal neurons in the hippocampus via its receptor (Lin et al. 2017). Stimulation of neurogenesis in the hippocampus by oxytocin has also been observed in other previous studies (Leuner et al. 2012). One study has suggested that neonatal application of oxytocin can affect serotonin axon length densities in the brain of male prairie voles (Eaton et al. 2012). Even so, neonatal manipulations of oxytocin can induce sexually dimorphic, age-dependent and site-specific alterations in the cells producing oxytocin itself (Yamamoto et al. 2004). Therefore, these authors especially stressed the development of the oxytocinergic system in the context of social behavior and the formation of pair bonds. Indeed, it has been proved repeatedly that oxy tocin belongs to the crucial factors responsible for the establishment of the social bonds and the regulation of the social behavior.

Recent studies have associated oxytocin with the perception of the social clues (Liu et al. 2018) and recognition of the adult faces in parent-child interaction (Peltola et al. 2018). The basis of these oxytocin effects could lie in the short-term effects of oxytocin on neuronal activity and neurotransmission, rather than the permanent structural consequences. Moreover, the short-term effects of oxytocin on GABA-ergic synapses have also been observed (Theodosis et al. 2006). Oxytocin receptors are associated with timing of the switch of the GABAergic neurotransmission from excitatory to inhibitory during the development of the nervous system (Leonzino et al. 2016). This concept remains to be further investigated to find out the extent of the oxytocin effect on the neuronal activity and/or neuronal morphology.

\section{Acknowledgment}

The work was supported by the projects $2 / 0038 / 18$ and 2/0116/16 of the Grant Agency of Ministry of Education and Slovak Academy of Sciences (VEGA) and by the Slovak Research and Development Agency project APVV-15-205 and APVV-15-0045. 


\section{References}

Abell F, Krams M, Ashburner J, Passingham R, Friston K, Frackowiak R, Happe F, Frith C, Frith U. The neuroanatomy of autism: a voxel-based whole brain analysis of structural scans. Neuroreport 10, 1647-1651, 1999.

Amaral DG, Schumann CM, Nordahl CW. Neuroanatomy of autism. Trends Neurosci 31, 137-145, 2008.

Auzias G, Viellard M, Takerkart S, Villeneuve N, Poinso F, Fonseca DD, Girard N, Deruelle C. Atypical sulcal anatomy in young children with autism spectrum disorder. Neuroimage Clin 4, 593-603, 2014.

Avino TA, Barger N, Vargas MV, Carlson EL, Amaral DG, Bauman MD, Schumann CM. Neuron numbers increase in the human amygdala from birth to adulthood, but not in autism. Proc Natl Acad Sci U S A 115, 3710-3715, 2018.

Bakos J, Bacova Z, Grant SG, Castejon AM, Ostatnikova D. Are molecules involved in neuritogenesis and axon guidance related to autism pathogenesis? Neuromolecular Med 17, 297-304, 2015.

Bakos J, Zatkova M, Bacova Z, Ostatnikova D. The role of hypothalamic neuropeptides in neurogenesis and neuritogenesis. Neural Plast 2016, 3276383, 2016.

Baron-Cohen S, Ring HA, Bullmore ET, Wheelwright S, Ashwin C, Williams SC. The amygdala theory of autism. Neurosci Biobehav Rev 24, 355-364, 2000.

Baron-Mendoza I, Garcia O, Calvo-Ochoa E, Rebollar-Garcia JO, Garzon-Cortes D, Haro R, Gonzalez-Arenas A. Alterations in neuronal cytoskeletal and astrocytic proteins content in the brain of the autistic-like mouse strain C58/J. Neurosci Lett 682, 32-38, 2018.

Bellani M, Calderoni S, Muratori F, Brambilla P. Brain anatomy of autism spectrum disorders I. Focus on corpus callosum. Epidemiol Psychiatr Sci 22, 217-221, 2013a.

Bellani M, Calderoni S, Muratori F, Brambilla P. Brain anatomy of autism spectrum disorders II. Focus on amygdala. Epidemiol Psychiatr Sci 22, 309-312. 2013b.

Blackmon K, Ben-Avi E, Wang X, Pardoe HR, Di Martino A, Halgren E, Devinsky O, Thesen T, Kuzniecky R. Periventricular white matter abnormalities and restricted repetitive behavior in autism spectrum disorder. Neuroimage Clin 10, 36-45, 2015.

Bozdagi O, Sakurai T, Papapetrou D, Wang X, Dickstein DL, Takahashi N, Kajiwara Y, Yang M, Katz AM, Scattoni ML, Harris MJ, Saxena R, Silverman JL, Crawley JN, Zhou Q, Hof PR, Buxbaum JD. Haploinsufficiency of the autism-associated Shank3 gene leads to deficits in synaptic function, social interaction, and social communication. Mol Autism 1, 15, 2010.

Brambilla P, Hardan A, di Nemi SU, Perez J, Soares JC, Barale F. Brain anatomy and development in autism: review of structural MRI studies. Brain Res Bull 61, 557-569, 2003.

Carper RA, Courchesne E. Localized enlargement of the frontal cortex in early autism. Biol Psychiatry 57, 126-133, 2005.

Chapman DB, Theodosis DT, Montagnese C, Poulain DA, Morris JF. Osmotic stimulation causes structural plasticity of neurone-glia relationships of the oxytocin but not vasopressin secreting neurones in the hypothalamic supraoptic nucleus. Neuroscience 17, 679-686, 1986.

Comin $\mathrm{CH}$, da Fontoura Costa L. Shape, connectedness and dynamics in neuronal networks. J Neurosci Methods 220, 100-115, 2013.

Courchesne E, Karns CM, Davis HR, Ziccardi R, Carper RA, Tigue ZD, Chisum HJ, Moses P, Pierce K, Lord C, Lincoln AJ, Pizzo S, Schreibman L, Haas RH, Akshoomoff NA, Courchesne RY. Unusual brain growth patterns in early life in patients with autistic disorder: an MRI study. Neurology 57, 245-254, 2001.

Courchesne E, Campbell K, Solso S. Brain growth across the life span in autism: age-specific changes in anatomical pathology. Brain Res 1380, 138-145, 2011a.

Courchesne E, Mouton PR, Calhoun ME, Semendeferi K, Ahrens-Barbeau C, Hallet MJ, Barnes CC, Pierce K. Neuron number and size in prefrontal cortex of children with autism. JAMA 306, 2001-2010, 2011 b.

Crawford JD, Chandley MJ, Szebeni K, Szebeni A, Waters B, Ordway GA. Elevated GFAP Protein in Anterior Cingulate Cortical White Matter in Males With Autism Spectrum Disorder. Autism Res 8, 649-657, 2015.

DeRamus TP, Kana RK. Anatomical likelihood estimation meta-analysis of grey and white matter anomalies in autism spectrum disorders. Neuroimage Clin 7, 525-536, 2014.

Donovan AP, Basson MA. The neuroanatomy of autism - a developmental perspective. J Anat 230, 4-15, 2017.

Duffney LJ, Zhong P, Wei J, Matas E, Cheng J, Qin L, Ma K, Dietz DM, Kajiwara Y, Buxbaum JD, Yan Z. Autism-like deficits in shank3-deficient mice are rescued by targeting actin regulators. Cell Rep 11, 1400-1413, 2015.

Eaton JL, Roache L, Nguyen KN, Cushing BS, Troyer E, Papademetriou E, Raghanti MA. Organizational effects of oxytocin on serotonin innervation. Dev Psychobiol 54, 92-97, 2012. 
Ecker C, Bookheimer SY, Murphy DG. Neuroimaging in autism spectrum disorder: brain structure and function across the lifespan. Lancet Neurol 14, 1121-1134, 2015.

Gallese V. The roots of empathy: the shared manifold hypothesis and the neural basis of intersubjectivity. Psychopathology 36, 171-180, 2003.

Gillberg CL. The Emanuel Miller Memorial Lecture 1991. Autism and autistic-like conditions: subclasses among disorders of empathy. J Child Psychol Psychiatry 33, 813-842, 1992.

Gordon-Weeks PR, Fournier AE. Neuronal cytoskeleton in synaptic plasticity and regeneration. J Neurochem 129, 206-212, 2014.

Haar S, Berman S, Behrmann M, Dinstein I. Anatomical abnormalities in autism? Cereb Cortex 26, 1440-1452, 2016.

Hadjikhani N, Joseph RM, Snyder J, Tager-Flusberg H. Anatomical differences in the mirror neuron system and social cognition network in autism. Cereb Cortex 16, 1276-1282, 2006.

Hruska M, Henderson N, Le Marchand SJ, Jafri H, Dalva MB. Synaptic nanomodules underlie the organization and plasticity of spine synapses. Nat Neurosci 21, 671-682, 2018.

Joensuu M, Lanoue V, Hotulainen P. Dendritic spine actin cytoskeleton in autism spectrum disorder. Prog Neuropsychopharmacol Biol Psychiatry 84, 362-381, 2018.

Kaufmann WE, Cooper KL, Mostofsky SH, Capone GT, Kates WR, Newschaffer CJ, Bukelis I, Stump MH, Jann AE, Lanham DC. Specificity of cerebellar vermian abnormalities in autism: a quantitative magnetic resonance imaging study. J Child Neurol 18, 463-470, 2003.

Kucharsky Hiess R, Alter R, Sojoudi S, Ardekani BA, Kuzniecky R, Pardoe HR. Corpus callosum area and brain volume in autism spectrum disorder: quantitative analysis of structural MRI from the ABIDE database. J Autism Dev Disord 45, 3107-3114, 2015.

Lee TT, Skafidas E, Dottori M, Zantomio D, Pantelis C, Everall I, Chana G. No preliminary evidence of differences in astrocyte density within the white matter of the dorsolateral prefrontal cortex in autism. Mol Autism 8, 64, 2017.

Leonzino M, Busnelli M, Antonucci F, Verderio C, Mazzanti M, Chini B. The timing of the excitatory-to-inhibitory GABA switch is regulated by the oxytocin receptor via KCC2. Cell Rep 15, 96-103, 2016.

Leslie KR, Johnson-Frey SH, Grafton ST. Functional imaging of face and hand imitation: towards a motor theory of empathy. Neuroimage 21, 601-607, 2004.

Lestanova Z, Bacova Z, Kiss A, Havranek T, Strbak V, Bakos J. Oxytocin increases neurite length and expression of cytoskeletal proteins associated with neuronal growth. J Mol Neurosci 59, 184-192, 2016.

Leuner B, Caponiti JM, Gould E. Oxytocin stimulates adult neurogenesis even under conditions of stress and elevated glucocorticoids. Hippocampus 22, 861-868, 2012.

Lin YT, Chen CC, Huang CC, Nishimori K, Hsu KS. Oxytocin stimulates hippocampal neurogenesis via oxytocin receptor expressed in CA3 pyramidal neurons. Nat Commun 8, 537, 2017.

Liu R, Yuan X, Chen K, Jiang Y, Zhou W. Perception of social interaction compresses subjective duration in an oxytocin-dependent manner. Elife 7, pii: e32100, 2018.

McAlonan GM, Daly E, Kumari V, Critchley HD, van Amelsvoort T, Suckling J, Simmons A, Sigmundsson T, Greenwood K, Russell A, Schmitz N, Happe F, Howlin P, Murphy DG. Brain anatomy and sensorimotor gating in Asperger's syndrome. Brain 125, 1594-1606, 2002.

Meyer M, Berger I, Winter J, Jurek B. Oxytocin alters the morphology of hypothalamic neurons via the transcription factor myocyte enhancer factor 2A (MEF-2A). Mol Cell Endocrinol 477, 156-162, 2018.

Nagel J, Delandre C, Zhang Y, Forstner F, Moore AW, Tavosanis G. Fascin controls neuronal class-specific dendrite arbor morphology. Development 139, 2999-3009, 2012.

Nickl-Jockschat T, Habel U, Michel TM, Manning J, Laird AR, Fox PT, Schneider F, Eickhoff SB. Brain structure anomalies in autism spectrum disorder--a meta-analysis of VBM studies using anatomic likelihood estimation. Hum Brain Mapp 33, 1470-1489, 2012.

Palanisamy A, Kannappan R, Xu Z, Martino A, Friese MB, Boyd JD, Crosby G, Culley DJ. Oxytocin alters cell fate selection of rat neural progenitor cells in vitro. PLoS One 13, e0191160, 2018.

Pani G, Samari N, Quintens R, de Saint-Georges L, Meloni M, Baatout S, Van Oostveldt P, Benotmane MA. Morphological and physiological changes in mature in vitro neuronal networks towards exposure to short-, middleor long-term simulated microgravity. PLoS One 8, e73857, 2013.

Paul LK, Brown WS, Adolphs R, Tyszka JM, Richards LJ, Mukherjee P, Sherr EH. Agenesis of the corpus callosum: genetic, developmental and functional aspects of connectivity. Nat Rev Neurosci 8, 287-299, 2007.

Pearson BL, Corley MJ, Vasconcellos A, Blanchard DC, Blanchard RJ. Heparan sulfate deficiency in autistic postmortem brain tissue from the subventricular zone of the lateral ventricles. Behav Brain Res 243, 138-145, 2013. 
Peltola MJ, Strathearn L, Puura K. Oxytocin promotes face-sensitive neural responses to infant and adult faces in mothers. Psychoneuroendocrinology 91, 261-270, 2018.

Peng H, Hawrylycz M, Roskams J, Hill S, Spruston N, Meijering E, Ascoli GA. BigNeuron: Large-Scale 3D Neuron Reconstruction from Optical Microscopy Images. Neuron 87, 252-256, 2015.

Piven J, Berthier ML, Starkstein SE, Nehme E, Pearlson G, Folstein S. Magnetic resonance imaging evidence for a defect of cerebral cortical development in autism. Am J Psychiatry 147, 734-739, 1990.

Rapin I, Katzman R. Neurobiology of autism. Ann Neurol 43, 7-14, 1998.

Raymond GV, Bauman ML, Kemper TL. Hippocampus in autism: a Golgi analysis. Acta Neuropathol 91, 117-119, 1996.

Reichova A, Zatkova M, Bacova Z, Bakos J. Abnormalities in interactions of Rho GTPases with scaffolding proteins contribute to neurodevelopmental disorders. J Neurosci Res 96, 781-788, 2018.

Sadybekov A, Tian C, Arnesano C, Katritch V, Herring BE. An autism spectrum disorder-related de novo mutation hotspot discovered in the GEF1 domain of Trio. Nat Commun 8, 601, 2017.

Schumann CM, Barnes CC, Lord C, Courchesne E. Amygdala enlargement in toddlers with autism related to severity of social and communication impairments. Biol Psychiatry 66, 942-949, 2009.

Schumann CM, Nordahl CW. Bridging the gap between MRI and postmortem research in autism. Brain Res 1380, 175-186, 2011.

Stanfield AC, McIntosh AM, Spencer MD, Philip R, Gaur S, Lawrie SM. Towards a neuroanatomy of autism: a systematic review and meta-analysis of structural magnetic resonance imaging studies. Eur Psychiatry 23, 289-299, 2008.

Star EN, Kwiatkowski DJ, Murthy VN. Rapid turnover of actin in dendritic spines and its regulation by activity. Nat Neurosci 5, 239-246, 2002.

Suren P, Stoltenberg C, Bresnahan M, Hirtz D, Lie KK, Lipkin WI, Magnus P, Reichborn-Kjennerud T, Schjolberg S, Susser E, Oyen AS, Li L, Hornig M. Early growth patterns in children with autism. Epidemiology 24, 660-670, 2013.

Theodosis DT, Poulain DA. Evidence that oxytocin-secreting neurones are involved in the ultrastructural reorganisation of the rat supraoptic nucleus apparent at lactation. Cell Tissue Res 235, 217-219, 1984.

Theodosis DT, Koksma JJ, Trailin A, Langle SL, Piet R, Lodder JC, Timmerman J, Mansvelder H, Poulain DA, Oliet $\mathrm{SH}$, Brussaard AB. Oxytocin and estrogen promote rapid formation of functional GABA synapses in the adult supraoptic nucleus. Mol Cell Neurosci 31, 785-794, 2006.

Traut N, Beggiato A, Bourgeron T, Delorme R, Rondi-Reig L, Paradis AL, Toro R. Cerebellar volume in autism: Literature meta-analysis and analysis of the autism brain imaging data exchange cohort. Biol Psychiatry 83, 579-588, 2018.

Van Goor D, Hyland C, Schaefer AW, Forscher P. The role of actin turnover in retrograde actin network flow in neuronal growth cones. PLoS One 7, e30959, 2012.

Verkuyl JM, Matus A. Time-lapse imaging of dendritic spines in vitro. Nat Protoc 1, 2399-2405, 2006.

Wang YF, Hatton GI. Mechanisms underlying oxytocin-induced excitation of supraoptic neurons: prostaglandin mediation of actin polymerization. J Neurophysiol 95, 3933-3947, 2006.

Wang YF, Hatton GI. Interaction of extracellular signal-regulated protein kinase 1/2 with actin cytoskeleton in supraoptic oxytocin neurons and astrocytes: role in burst firing. J Neurosci 27, 13822-13834, 2007.

Wegiel J, Flory M, Kuchna I, Nowicki K, Ma SY, Imaki H, Wegiel J, Cohen IL, London E, Brown WT, Wisniewski T. Brain-region-specific alterations of the trajectories of neuronal volume growth throughout the lifespan in autism. Acta Neuropathol Commun 2, 28, 2014.

Yamamoto Y, Cushing BS, Kramer KM, Epperson PD, Hoffman GE, Carter CS. Neonatal manipulations of oxytocin alter expression of oxytocin and vasopressin immunoreactive cells in the paraventricular nucleus of the hypothalamus in a gender-specific manner. Neuroscience 125, 947-955, 2004.

Yang DY, Beam D, Pelphrey KA, Abdullahi S, Jou RJ. Cortical morphological markers in children with autism: a structural magnetic resonance imaging study of thickness, area, volume, and gyrification. Mol Autism 7, 11, 2016.

Zatkova M, Reichova A, Bacova Z, Strbak V, Kiss A, Bakos J. Neurite outgrowth stimulated by oxytocin is modulated by inhibition of the calcium voltage-gated channels. Cell Mol Neurobiol 38, 371-378, 2018.

Zeidan-Chulia F, Rybarczyk-Filho JL, Salmina AB, de Oliveira BH, Noda M, Moreira JC. Exploring the multifactorial nature of autism through computational systems biology: calcium and the Rho GTPase RAC1 under the spotlight. Neuromolecular Med 15, 364-383, 2013. 
Zielinski BA, Prigge MB, Nielsen JA, Froehlich AL, Abildskov TJ, Anderson JS, Fletcher PT, Zygmunt KM, Travers BG, Lange N, Alexander AL, Bigler ED, Lainhart JE. Longitudinal changes in cortical thickness in autism and typical development. Brain 137, 1799-1812, 2014. 